\title{
Automatic Image Enhancement by Content Dependent Exposure Correction
}

\author{
S. Battiato \\ University of Catania, Department of Mathematic and Informatics, 95125 Catania, Italy \\ Email:battiato@dmi.unict.it
}

A. Bosco

STMicroelectronics, M6 Site, Zona Industriale, 95121 Catania, Italy

Email: angelo.bosco@st.com

\section{A. Castorina}

STMicroelectronics, M6 Site, Zona Industriale, 95121 Catania, Italy

Email: alfio.castorina@st.com

\section{G. Messina}

STMicroelectronics, M6 Site, Zona Industriale, 95121 Catania, Italy

Email: giuseppe.messina@st.com

Received 7 August 2003; Revised 8 March 2004

\begin{abstract}
We describe an automatic image enhancement technique based on features extraction methods. The approach takes into account images in Bayer data format, captured using a CCD/CMOS sensor and/or 24-bit color images; after identifying the visually significant features, the algorithm adjusts the exposure level using a "camera response"-like function; then a final HUE reconstruction is achieved. This method is suitable for handset devices acquisition systems (e.g., mobile phones, PDA, etc.). The process is also suitable to solve some of the typical drawbacks due to several factors such as poor optics, absence of flashgun, and so forth.
\end{abstract}

Keywords and phrases: Bayer pattern, skin recognition, features extraction, contrast, focus, exposure correction.

\section{INTRODUCTION}

Reduction of processing time and quality enhancement of acquired images is becoming much more significant. The use of sensors with greater resolution combined with advanced solutions $[1,2,3,4]$ aims to improve the quality of resulting images. One of the main problems affecting image quality, leading to unpleasant pictures, comes from improper exposure to light. Beside the sophisticated features incorporated in today's cameras (i.e., automatic gain control algorithms), failures are not unlikely to occur. Some techniques are completely automatic, cases in point being represented by those based on "average/automatic exposure metering" or the more complex "matrix/intelligent exposure metering." Others, again, accord the photographer a certain control over the selection of the exposure, thus allowing space for personal taste or enabling him to satisfy particular needs.

Inspite of the great variety of methods $[5,6]$, for regulating the exposure and the complexity of some of them, it is not rare for images to be acquired with a nonoptimal or incorrect exposure. This is particularly true for handset devices (e.g., mobile phones) where several factors contribute to acquire bad-exposed pictures: poor optics, absence of flashgun, not to talk about "difficult" input scene lighting conditions, and so forth.

There is no exact definition of what a correct exposure should be. It is possible to abstract a generalization and to define the best exposure that enables one to reproduce the most important regions (according to contextual or perceptive criteria) with a level of gray or brightness, more or less in the middle of the possible range.

Using postprocessing techniques an effective enhancement should be obtained. Typical histogram specification, histogram equalization, and gamma correction to improve global contrast appearance [7] only stretch the global distribution of the intensity. More adaptive criterions are needed to overcome such drawback. In $[8,9]$ two adaptive histogram equalization techniques, able to modify intensity's 


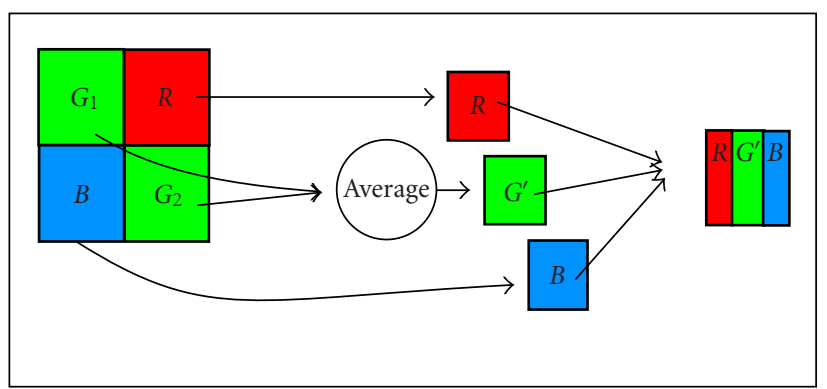

Figure 1: Bayer data subsampling generation.

distribution inside small regions are presented. In particular the method described in [9], splits the input image into two or more equal area subimages based on its gray-level probability density function. After having equalized each subimage, the enhanced image is built taking into account some local property, preserving the original image's average luminance. In [10] point processing and spatial filtering are combined together while in [11] a fuzzy logic approach to contrast enhancement is presented. Recent approaches work in the compressed domain [12] or use advanced techniques such as curvelet transform [13], although both methods are not suited for real-time processing.

The new exposure correction technique described in this paper is designed essentially for mobile sensors applications. This new element, present in newest mobile devices, is particularly harmed by "backlight" when the user utilizes a mobile device for video phoning. The detection of skin characteristics in captured images allows selection and proper enhancement and/or tracking of regions of interest (e.g., faces). If no skin is present in the scene the algorithm switches automatically to other features (such as contrast and focus) tracking for visually relevant regions. This implementation differs from the algorithm described in [14] because the whole processing can also be performed directly on Bayer pattern images [15], and simpler statistical measures were used to identify information carrying regions; furthermore the skin feature has been added.

The paper is organized as follows. Section 2 describes the different features extraction approaches and the exposure correction technique used for automatic enhancement. The "arithmetic" complexity [16] of the whole process is estimated in Section 3. In Section 4 experimental results show the effectiveness of the proposed techniques. Also some comparisons with other techniques $[7,9]$ are reported. Section 5 closes the paper tracking directions for future works.

\section{APPROACH DESCRIPTION}

The proposed automatic exposure correction algorithm is defined as follows.

(1) Luminance extraction. If the algorithm is applied on Bayer data, in place of the three full color planes, a subsampled (quarter size) approximated input data (see Figure 1) is used.
(2) Using a suitable features extraction technique the algorithm fixes a value to each region. This operation permits to seek visually relevant regions (for contrast and focus the regions are block-based, for skin recognition the regions are associated to each pixel).

(3) Once the "visually important" pixels are identified (e.g., the pixels belonging to skin features) a global tone correction technique is applied using as main parameter the mean gray levels of the relevant regions.

\subsection{Features extraction: contrast and focus}

To be able to identify regions of the image that contain more information, the luminance plane is subdivided in $N$ blocks of equal dimensions (in our experiments we employed $N=$ 64 for VGA images). For each block, statistical measures of "contrast" and "focus" are computed. Therefore it is assumed that well-focused or high-contrast blocks are more relevant compared to the others. Contrast refers to the range of tones present in the image. A high contrast leads to a higher number of perceptual significance regions inside a block.

Focus characterizes the sharpness or edgeness of the block and is useful in identifying regions where highfrequency components (i.e., details) are present.

If the aforementioned measures were simply computed on highly underexposed images, then the regions having better exposure would always have higher contrast and edgeness compared to those that are obscured. In order to perform a visual analysis revealing the most important features regardless of lighting conditions, a new "visibility image" is constructed by pushing the mean gray level of the input green Bayer pattern plane (or the $Y$ channel for color images) to 128. The push operation is performed using the same function that is used to adjust the exposure level and it will be described later.

The contrast measure is computed by simply building a histogram for each block and then calculating its deviation (2) from the mean value (3). A high deviation value denotes good contrast and vice versa. In order to remove irrelevant peaks, the histogram is slightly smoothed by replacing each entry with its mean in a ray 2 neighborhood. Thus, the original histogram entry is replaced with the gray level $\tilde{I}[i]$ :

$$
\tilde{I}[i]=\frac{(I[i-2]+I[i-1]+I[i]+I[i+1]+I[i+2])}{5} .
$$

Histogram deviation $D$ is computed as

$$
D=\frac{\sum_{i=0}^{255}|i-M| \cdot \tilde{I}[i]}{\sum_{i=0}^{255} \tilde{I}[i]},
$$

where $M$ is the mean value:

$$
M=\frac{\sum_{i=0}^{255} i \cdot \tilde{I}[i]}{\sum_{i=0}^{255} \tilde{I}[i]} .
$$

The focus measure is computed by convolving each block with a simple $3 \times 3$ Laplacian filter.

In order to discard irrelevant high-frequency pixels (mostly noise), the outputs of the convolution at each pixel 


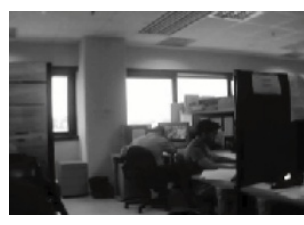

(a)

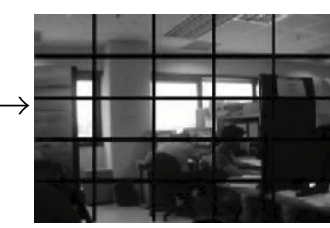

(b)

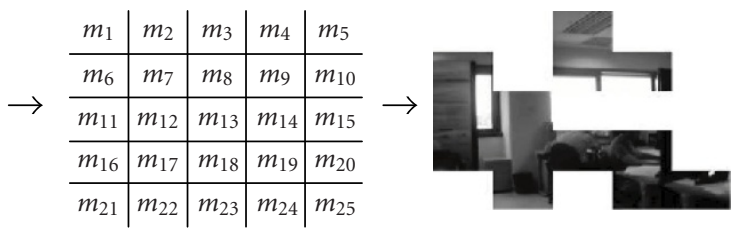

(c)

(d)

FIGURE 2: Features extraction pipeline (for focus and contrast with $N=25$ ). Visual relevance of each luminance block (b) of the input image (a) is based on relevance measures (c) able to obtain a list of relevant blocks (d).

are thresholded. The mean focus value of each block is computed as

$$
F=\frac{\sum_{i=1}^{N} \operatorname{thresh}[\operatorname{lapl}(i), \text { Noise }]}{N},
$$

where $N$ is the number of pixels and the thresh $(\cdot)$ operator discards values lower than a fixed threshold Noise. Once the values $F$ and $D$ are computed for all blocks, relevant regions will be classified using a linear combination of both values. Features extraction pipeline is illustrated in Figure 2.

\subsection{Features extraction: skin recognition}

As before a visibility image obtained by forcing the mean gray level of the luminance channel to be about 128 is built.

Most existing methods for skin color detection usually threshold some sort of measure of the likelihood of skin colors for each pixel and treat them independently. Human skin colors form a special category of colors, distinctive from the colors of most other natural objects. It has been found that human skin colors are clustered in various color spaces $[17,18]$. The skin color variations between people are mostly due to intensity differences. These variations can therefore be reduced by using chrominance components only.

Yang et al. [19] have demonstrated that the distribution of human skin colors can be represented by a twodimensional Gaussian function on the chrominance plane. The center of this distribution is determined by the mean vector $\vec{\mu}$ and its shape is determined by the covariance matrix $\Sigma$; both values can be estimated from an appropriate training data set. The conditional probability $p(\vec{x} \mid s)$ of a block belonging to the skin color class, given its chrominance vector $\vec{x}$ is then represented by

$$
p(\vec{x} \mid s)=\frac{1}{2 \pi}|\Sigma|^{-1 / 2} \exp \left\{\frac{-[d(\vec{x})]^{2}}{2}\right\},
$$

where $d(\vec{x})$ is the so-called Mahalanobis distance from the vector $\vec{x}$ to the mean vector $\vec{\mu}$ and is defined as

$$
[d(\vec{x})]^{2}=(\vec{x}-\vec{\mu})^{\prime} \Sigma^{-1}(\vec{x}-\vec{\mu}) .
$$

The value $d(\vec{x})$ determines the probability that a given block belongs to the skin color class. The larger the distance $d(\vec{x})$, the lower the probability that the block belongs to the skin color class $s$. Such class has been experimentally

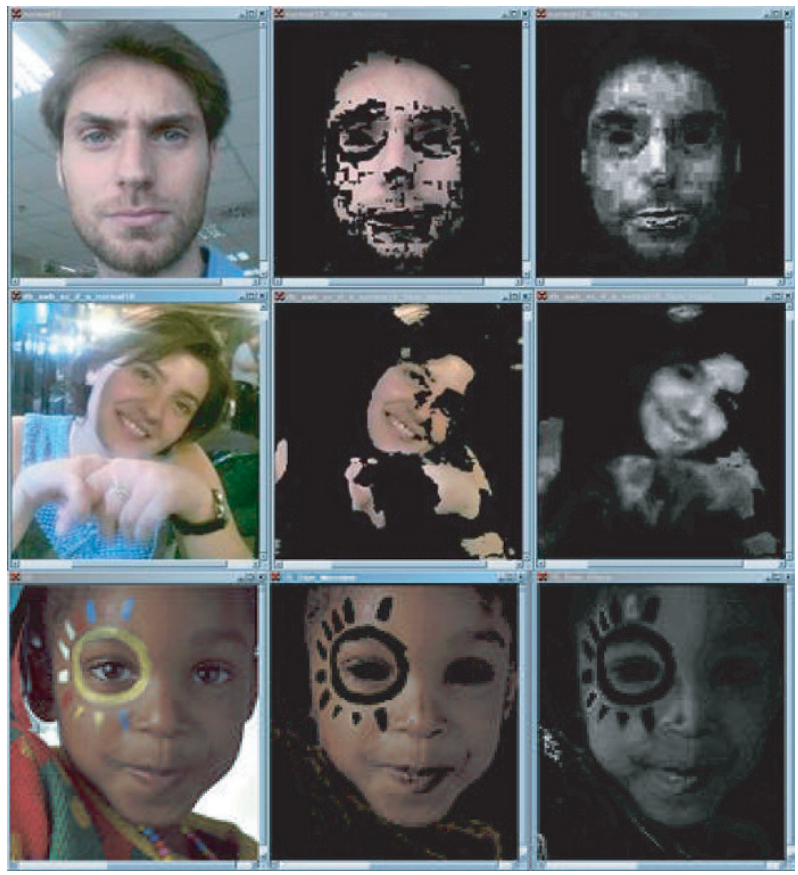

(a)

(b)

(c)

FIGURE 3: Skin recognition examples on RGB images: (a) original images acquired by Nokia 7650 phone (first and second row) with VGA sensor and compressed in JPEG format; (b) simplest threshold method output; and (c) probabilistic threshold output. Third image (a) is a standard test image.

derived using a large data set of images acquired at different conditions and resolution using CMOS-VGA sensor on "STV6500-E01" evaluation kit equipped with "502 VGA sensor" [20].

Due to the large quantity of color spaces, distance measures, and two-dimensional distributions, many skin recognition algorithms can be used. The skin color algorithm is independent of exposure correction, thus we introduce two different alternative techniques aimed to recognize skin regions (as shown in Figure 3).

(1) By using the input $\mathrm{YCbCr}$ image and the conditional probability (5), each pixel is classified as belonging to a skin region or not. Then a new image with normalized gray-scale values is derived, where skin areas are 


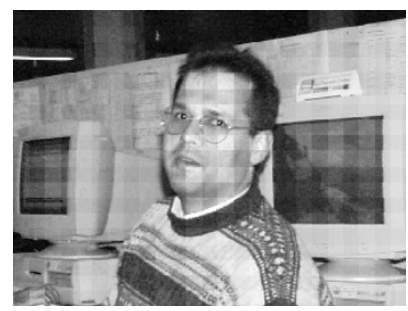

(a)

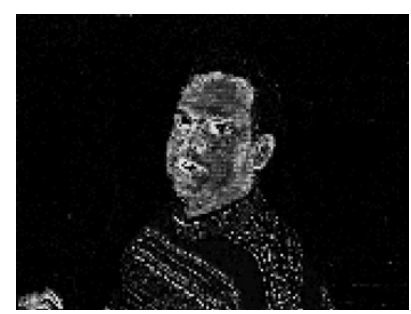

(b)

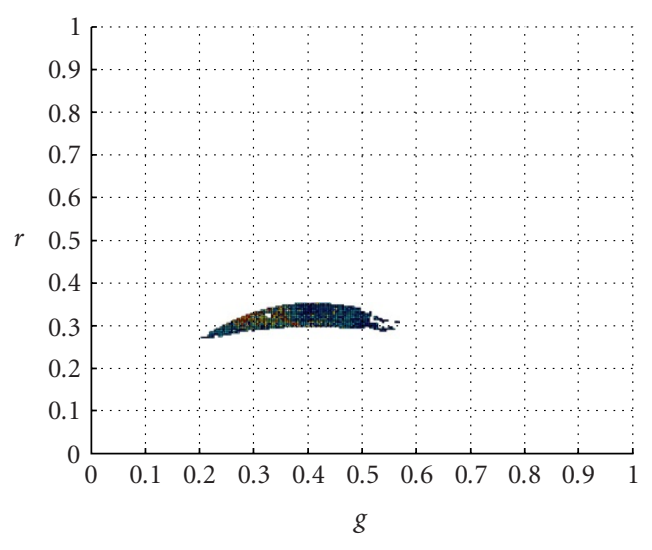

(c)

Figure 4: Skin recognition examples on Bayer pattern image: (a) original image in Bayer data; (b) recognized skin with probabilistic approach; and (c) threshold skin values on $r-g$ bidirectional histogram (skin locus).

properly highlighted (Figure $3 \mathrm{c}$ ). The higher the gray value the higher the probability to compute a reliable identification.

(2) By processing an input RGB image, a 2D chrominance distribution histogram $(r, g)$ is computed, where $r=$ $R /(R+G+B)$ and $g=G /(R+G+B)$. Chrominance values representing skin are clustered in a specific area of the $(r, g)$ plane, called "skin locus" (Figure 4c), as defined in [21]. Pixels having a chrominance value belonging to the skin locus will be selected to correct exposure.

For Bayer data, the skin recognition algorithm works on the RGB image created by subsampling the original picture, as described in Figure 1.

\subsection{Exposure correction}

Once the visually relevant regions are identified, the exposure correction is carried out using the mean gray value of those regions as reference point. A simulated camera response curve is used for this purpose, which gives an estimate of how light values falling on the sensor become final pixel values (see Figure 5). Thus it is a function:

$$
f(q)=I,
$$

where $q$ represents the "light" quantity and $I$ the final pixel

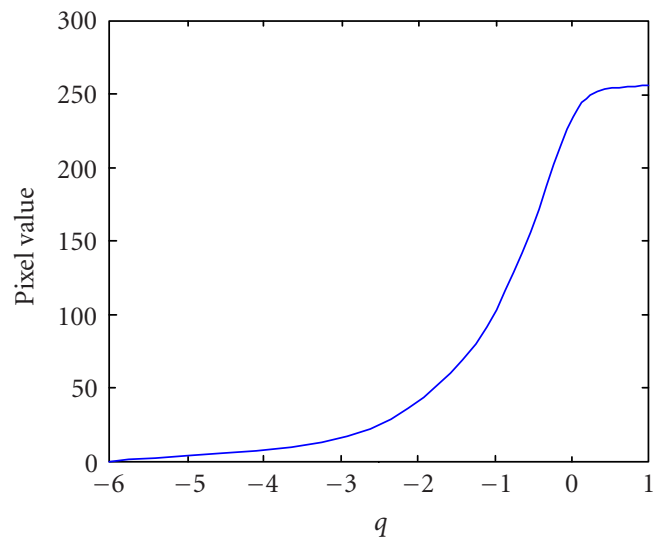

FIGURE 5: Simulated camera response.

value [1]. This function can be expressed $[14,22]$ by using a simple parametric closed form representation:

$$
f(q)=\frac{255}{\left(1+e^{-(A q)}\right)^{C}},
$$

where parameters $A$ and $C$ can be used to control the shape of the curve and $q$ is supposed to be expressed in 2-based logarithmic unit (usually referred as "stops"). These parameters could be estimated, depending on the specific image acquisition device, using the techniques described in [22] or chosen experimentally. The offset from the ideal exposure is computed using the $f$ curve and the average gray level of visually relevant regions avg as

$$
\Delta=f^{-1}(\operatorname{Trg})-f^{-1}(\operatorname{avg}),
$$

where $\operatorname{Trg}$ is the desired target gray level. Trg should be around 128 but its value could be slightly changed especially when dealing with Bayer pattern data where some postprocessing is often applied.

The luminance value $Y(x, y)$ of a pixel $(x, y)$ is modified as follows:

$$
Y^{\prime}(x, y)=f\left(f^{-1}(Y(x, y))+\Delta\right) .
$$

Note that all pixels are corrected. Basically the previous step is implemented as a lookup table (LUT) transform (Figure 6 shows two correction curves with different $A, C$ parameters). Final color reconstruction is done using the same approach described in [23] to prevent relevant HUE shifts and/or color desaturation:

$$
\begin{aligned}
& R^{\prime}=0.5 \cdot\left(\frac{Y^{\prime}}{Y} \cdot(R+Y)+R-Y\right), \\
& G^{\prime}=0.5 \cdot\left(\frac{Y^{\prime}}{Y} \cdot(G+Y)+G-Y\right), \\
& B^{\prime}=0.5 \cdot\left(\frac{Y^{\prime}}{Y} \cdot(B+Y)+B-Y\right),
\end{aligned}
$$

where $R, G$, and $B$ are the input color values.

Note that when Bayer pattern is used (10) is directly applied on RGB pixels. 


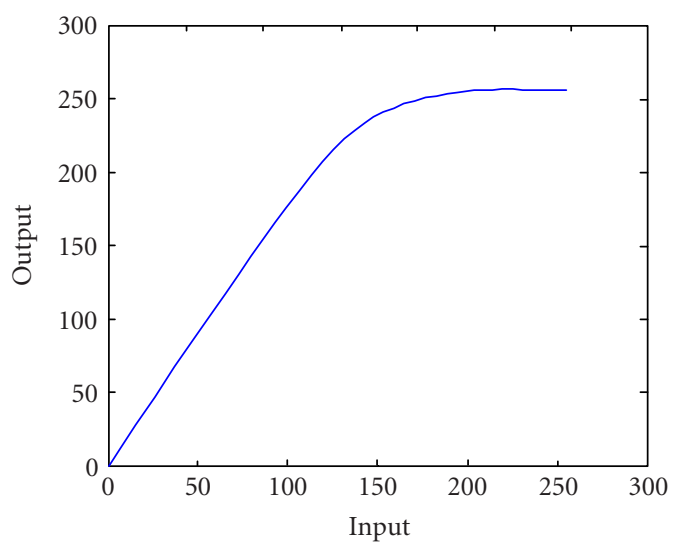

(a)

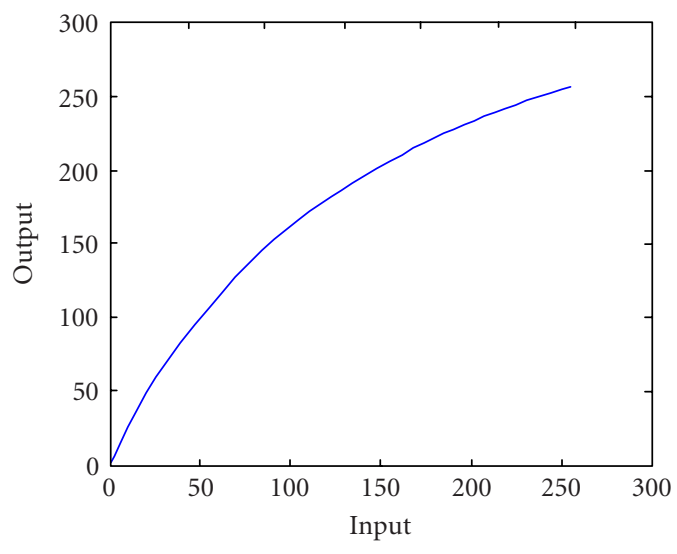

(b)

Figure 6: LUTs derived from curves with (a) $A=7$ and $C=0.13$ and (b) $A=0.85$ and $C=1$.

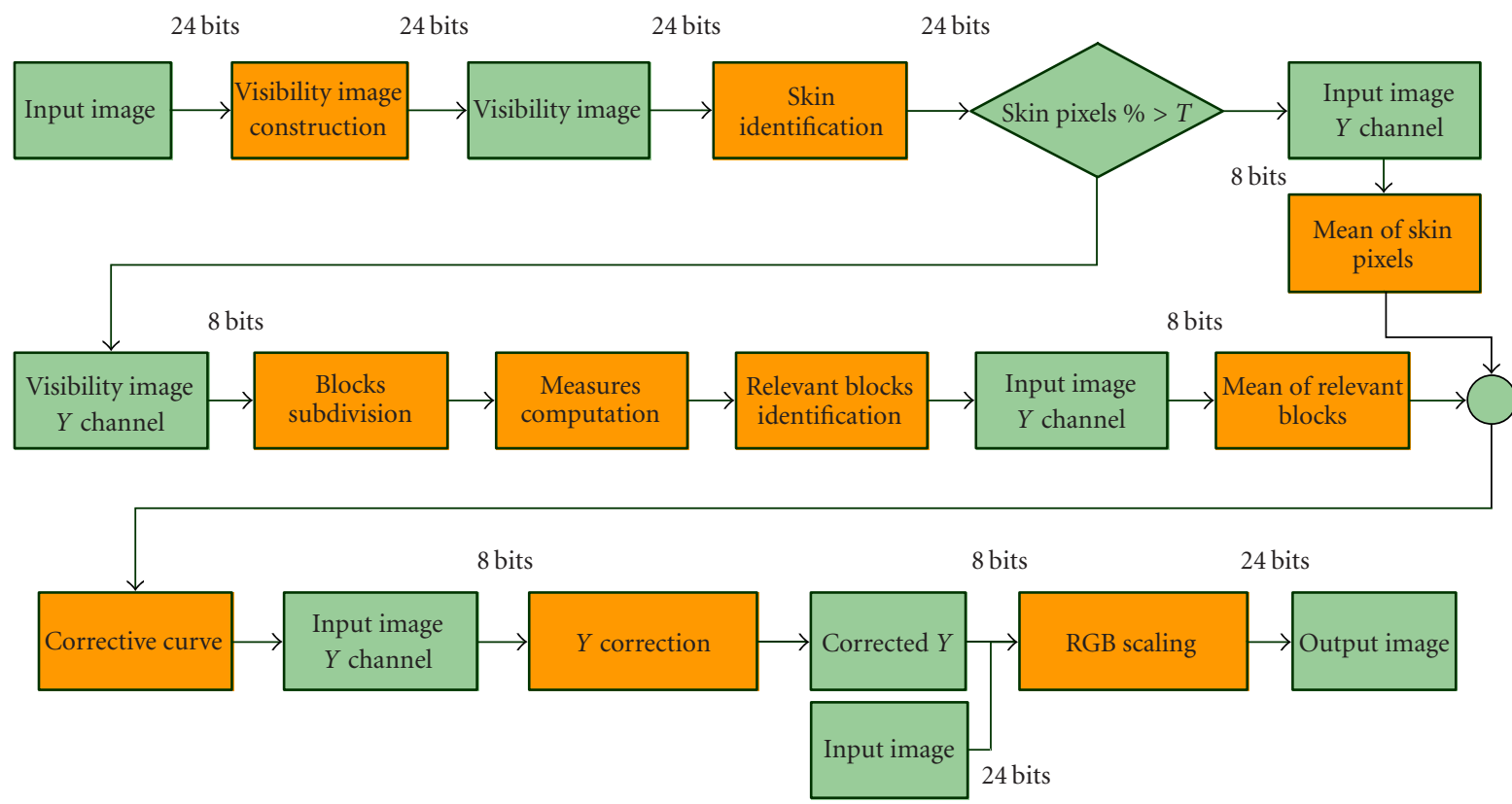

FIGURE 7: Automatic exposure correction pipeline: given a color image as input (for Bayer data image the pipeline is equivalent), the visibility image is extracted using a forced gray-level mean of about 128, then the skin percentage measure is achieved to seek if the input image contains skin features. In the case of skin feature existence (the value is more than the threshold $T$ ), the mean of selected skin pixel is achieved. If skin is not present the contrast and focus measures are computed and the mean of relevant blocks is performed. Finally, by fixing the correction curve, the exposure adjustment of luminance channel is accomplished.

\section{COMPLEXITY ANALYSIS}

The computational resources required by the algorithm described are negligible and indeed the whole process is well suited for real-time applications. Instead of the asymptotic complexity, the arithmetic complexity has been described to estimate the algorithm real-time execution [16]. More precisely, the sum of operations per pixel has been computed.

The following weights will be used:

(1) $w_{a}$ for basic arithmetic operations: additions, subtractions, comparisons, and so forth;
(2) $w_{m}$ for semicomplex arithmetic operations: multiplications, and so forth;

(3) $w_{l}$ for basic bits and logical operations: bits-shifts, logical operations, and so forth;

(4) $w_{c}$ for complex arithmetic operations: divisions, exponentials, and so forth.

First the main functions of the algorithm will be analyzed; then the overall $C$ complexity will be estimated.

A simple analysis of the computational cost can be carried out exploiting the main processing blocks composing the working flow of Figure 7 and considering the worst-case 
scenario, when the algorithm is applied directly on the RGB image. The following assumptions are considered:

(1) the image consists in $N \times M=$ tot pixels and $V \times H=$ num blocks;

(2) the inverse $f^{-1}$ of the $f$ function is stored in a 256element LUT;

(3) the value calculated by the function $f(10)$ is estimated by scanning the curve bottom-up (if $\Delta>0$ ) searching for the first LUT index $I$, where $\operatorname{LUT}[i]>\operatorname{LUT}[y]+$ $\Delta$, or top-down (if $\Delta<0$ ) searching for the first LUT index $i$ where LUT $[i]<\operatorname{LUT}[y]+\Delta$. In both cases $i$ becomes the value of gray-level $y$ after correction.

By using the above-mentioned assumptions the correction of the $Y$ channel can be done employing two 256-element LUTs, the first contains the $f^{-1}$ function and the second the outputs of (10) for each of the 256 possible gray levels. The second LUT can be initialized with a linear search for each gray level.

\section{Visibility image construction}

The visibility image is obtained by computing the mean of the extracted $Y$ and the offset from desired exposure by applying (9). Once the offset is known the visibility image is built using equations (10) to (13).

(1) Initialization step:

(a) mean computation: $1 w_{a}+(1 /$ tot $) w_{c}$;

(b) offset computation: (3/ tot) $w_{a}$;

(c) corrective curve uploading: $(2 k /$ tot $) w_{a}$, where $k$ has a mean value of about 70 in the worst case.

(2) Color correction:

$$
6 w_{a}+6 w_{m}+3 w_{c}
$$

Therefore

$$
C_{1}=\left(7+\frac{2 k+3}{\text { tot }}\right) w_{a}+6 w_{m}+\left(3+\frac{1}{\text { tot }}\right) w_{c}
$$

\section{Skin identification}

Since the skin probabilities are computed on $\mathrm{Cr}, \mathrm{Cb}$ channels defined in the 0-255 range (after the 128-offset addition) the probabilities for each possible $\mathrm{Cr}, \mathrm{Cb}$ pair can be precomputed and stored in a $256 \times 256$ LUT. The dimensions of this LUT, due to its particular shape (Figure 8), can be reduced up to $136 \times 86$ discarding the pairs having zero value:

(1) lookup of skin probabilities (simple access to LUT): $1 w_{a}$

(2) thresholding of skin probabilities: $1 w_{a}$;

(3) computation of skin mean gray value: $1 w_{a}+(1 /$ tot $) w_{c}$.

Therefore

$$
C_{2}=3 w_{a}+\left(\frac{1}{\text { tot }}\right) w_{c}
$$

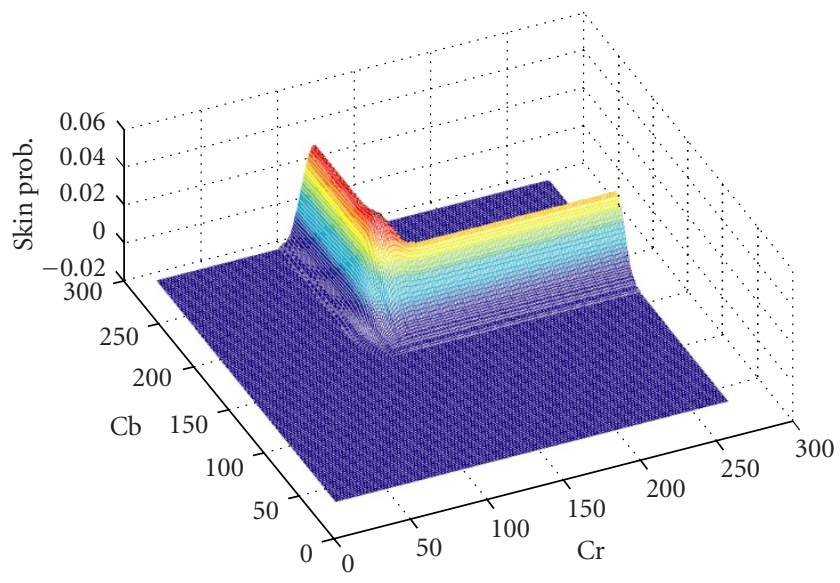

FIGURE 8: Skin precomputed LUT.

\section{Measures computation}

The mean, focus, and contrast of each block are computed.

(1) Mean values of each block: $\left(\right.$ num $\left.\times w_{c}\right) /$ tot (since accumulated gray levels inside each block can be obtained from the visibility image and only the divisions have to be done).

(2) Focus computation:

$$
\left(1 w_{l}+6 w_{a}\right)+1 w_{a}+\left(\frac{\text { num }}{\text { tot }}\right) w_{c}
$$

(3) Contrast computation:

$$
\frac{\left(256\left(11 w_{a}+w_{m}+w_{c}\right)+1 w_{c}\right) \text { num }}{\text { tot }} .
$$

Therefore:

$$
\begin{aligned}
C_{3}= & \left(7+2816 \frac{\mathrm{num}}{\mathrm{tot}}\right) w_{a}+w_{l} \\
& +\left(256 \frac{\mathrm{num}}{\mathrm{tot}}\right) w_{m}+\left(259 \frac{\mathrm{num}}{\mathrm{tot}}\right) w_{c} .
\end{aligned}
$$

\section{Relevant blocks identification}

Once focus and contrast are obtained, blocks are selected using their linear combination value:

(1) linear combination of focus and contrast: (num/ tot) $\left(1 w_{a}+2 w_{m}\right)$

(2) comparison between the linear combination and a selection value: (num/tot) $w_{m}$.

Therefore

$$
C_{4}=\left(\frac{\text { num }}{\text { tot }}\right)\left(1 w_{a}+3 w_{m}\right)
$$




\section{Image correction}

This step can be considered computationally equivalent to the visibility image construction since the only difference is the mean value used for corrective LUT loading, therefore:

$$
C_{5}=\left(7+\frac{2 k+3}{\text { tot }}\right) w_{a}+6 w_{m}+\left(3+\frac{1}{\text { tot }}\right) w_{c} .
$$

The algorithm complexity is then obtained by adding all the above values:

$$
\begin{aligned}
C= & \sum_{i=1}^{5} C_{i} \\
= & w_{l}+\left(21+\frac{4 k+6}{\text { tot }}+2817 \frac{\text { num }}{\text { tot }}\right) w_{a} \\
& +\left(12+259 \frac{\text { num }}{\text { tot }}\right) w_{m}+\left(6+\frac{2}{\text { tot }}+259 \frac{\text { num }}{\text { tot }}\right) w_{c} .
\end{aligned}
$$

The overall complexity is hence well suited for real-time applications (note that the ratio num/tot will always be very small, since tot $\gg$ num). For example given a $640 \times 480 \mathrm{VGA}$ input image ( tot $=307200)$, a fixed num $=64$ blocks, and the worst $k=70$, the complexity becomes

$$
\begin{aligned}
C=\sum_{i=1}^{5} C_{i}= & w_{l}+\left(21+\frac{76}{307200}+2817 \frac{64}{307200}\right) w_{a} \\
& +\left(12+259 \frac{64}{307200}\right) w_{m} \\
& +\left(6+\frac{2}{307200}+259 \frac{64}{307200}\right) w_{c} .
\end{aligned}
$$

Therefore

$$
C=\sum_{i=1}^{5} C_{i}=w_{l}+21.587 w_{a}+12.054 w_{m}+6.054 w_{c} .
$$

That is cost-effective and suitable for real-time processing applications.

\section{EXPERIMENTAL RESULTS}

The proposed technique has been tested using a large database of images acquired at different resolutions, with different acquisition devices, both in Bayer and RGB format. In Figure 7 the exposure correction pipeline is illustrated. The whole process is organized as follows: the "visibility" image is extracted from the input image, and then the skin percentage measure is achieved to determine if the input image contains skin features; once the type of features is known the extraction of the mean values is performed, and finally the correction is accomplished. In the Bayer case the algorithm was inserted in a real-time framework, using a CMOS-VGA sensor on STV6500-E01 evaluation kit equipped with 502 VGA sensor [20]. In Figure 9 screen shots of the working environ-

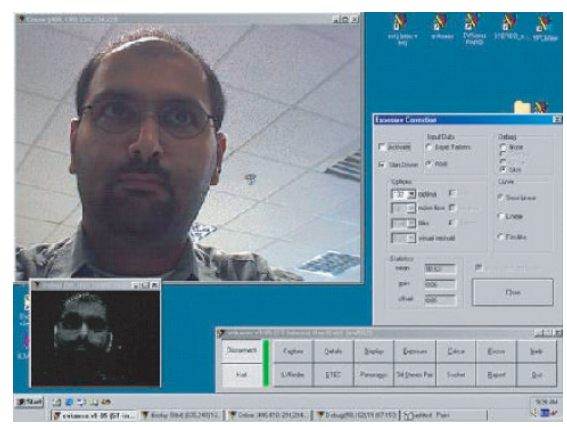

(a)

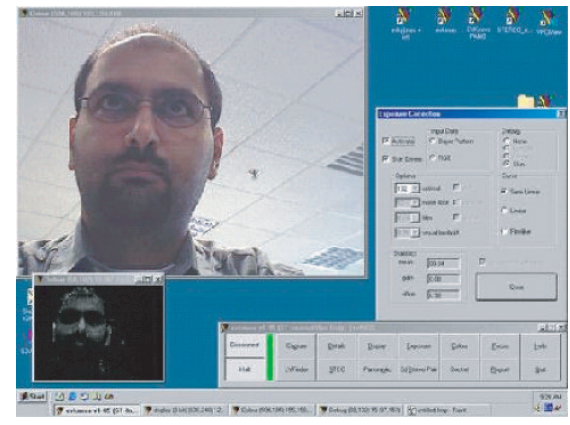

(b)

Figure 9: Framework interface for STV6500-E01 EVK 502 VGA sensor: (a) before and (b) during real-time skin dependent exposure correction. The small window with black background represents the detected skin.

ment are shown. Figure 10b illustrates the visually relevant blocks found during the features extraction step. Examples of skin detection by using real-time processing are reported in Figure 11. In the RGB case the algorithm could be implemented as postprocessing step. Examples of skin and contrast/focus exposure correction are respectively shown in Figures 10 and 12 .

For sake of comparisons we have chosen both global and adaptive techniques, able to work in real-time processing: standard global histogram equalization and gamma correction [7] and adaptive luminance preservation equalization technique [9]. The parameters of gamma correction have been manually fixed to the mean value computed by the proposed algorithm. Experiments and comparisons with existing methods are shown in Figures 13, 14, and 15.

In Figure 13a the selected image has been captured by using an Olympus C120 camera. It is evident that an overexposure is required. Both equalization algorithms in Figures 13b and $13 \mathrm{c}$ have introduced excessive contrast correction (the faces and the high frequencies of the two persons have been destroyed). The input image of Figure 14a has been captured by using an Olympus E10 camera. In this case the adaptive equalization algorithm in Figure $14 \mathrm{~b}$ has performed a better enhancement than in the previous example (Figure 13b), but the image still contains an excessive contrast correction (the face has lost skin luminance). The equalization in Figure 14c 

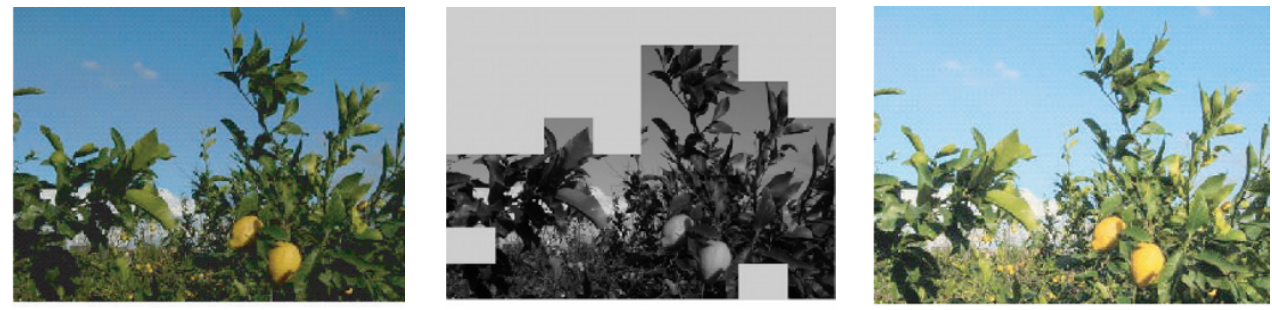

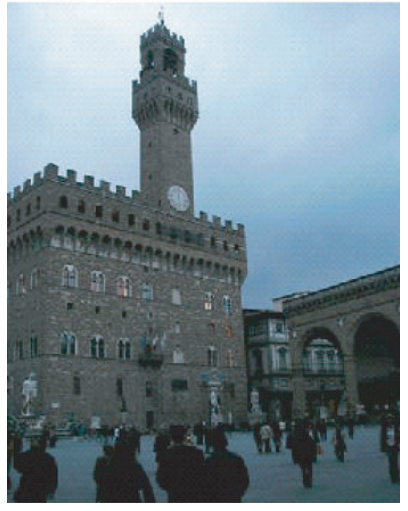

(a)

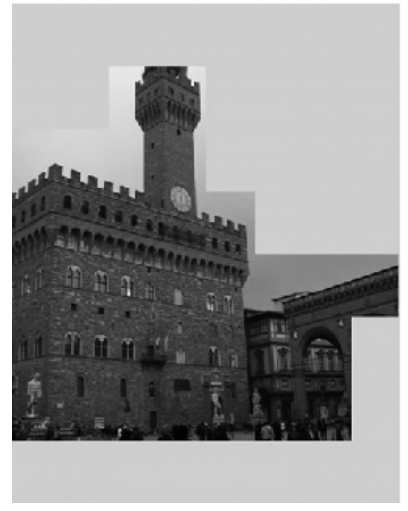

(b)

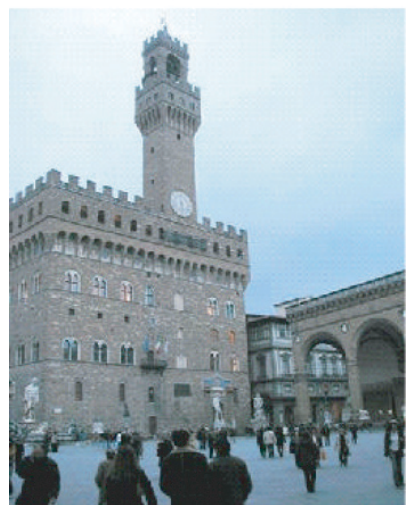

(c)

FIGURE 10: Experimental results by postprocessing: (a) original color input image, (b) contrast and focus visually significant blocks detected, and (c) exposure-corrected image obtained from RGB image.

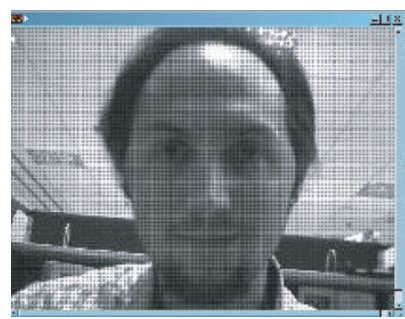

(a)

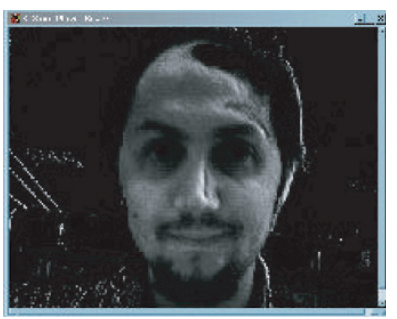

(b)

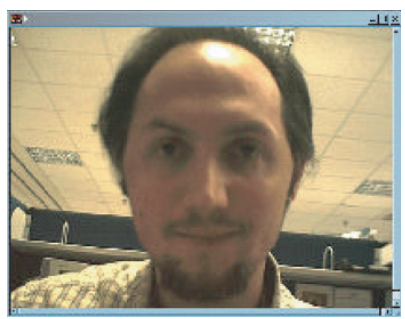

(c)

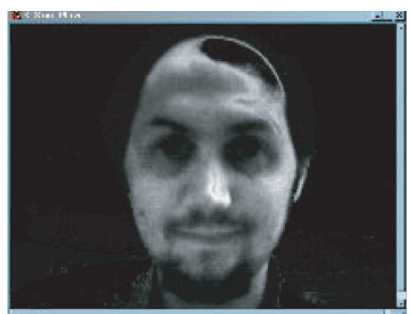

(d)

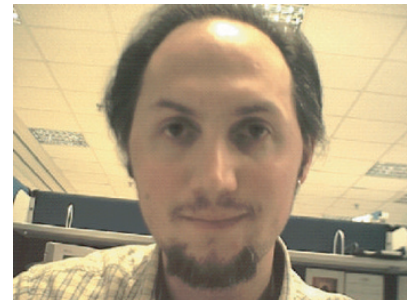

(e)

FIGURE 11: Experimental results by real-time and postprocessing: (a) original Bayer input image, (b) Bayer skin detected in real-time, (c) color interpolated image from Bayer input, (d) RGB skin detected in postprocessing, and (e) exposure-corrected image obtained from RGB image.

has completely failed the objective due to the large amount of background lightness. The exclusion of the skin features extraction phase is evident looking at the enhancement difference between Figures 14e and 14f. Finally, Figure 15 shows a poorly exposed image in Figure 15a acquired by using an Olympus C40Z camera. Both equalization algorithms Figures $15 \mathrm{~b}$ and $15 \mathrm{c}$ have introduced excessive contrast correction (the clouds and the grass are becoming darker). 


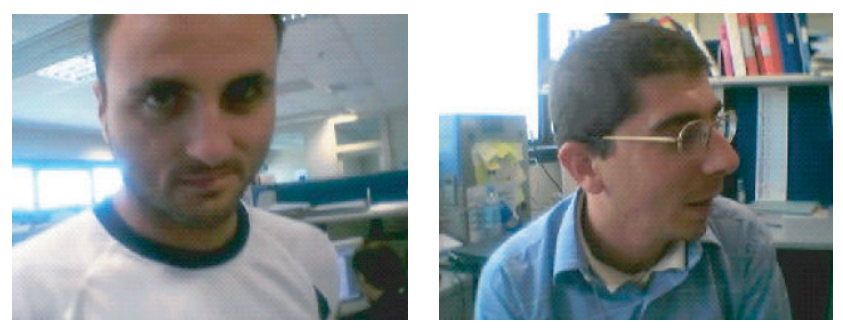

(a)

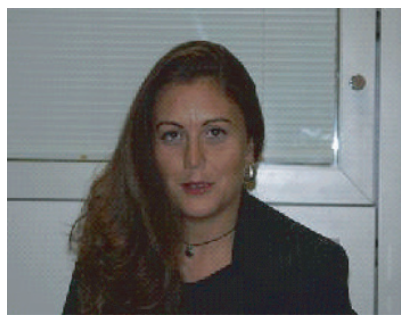

(c)
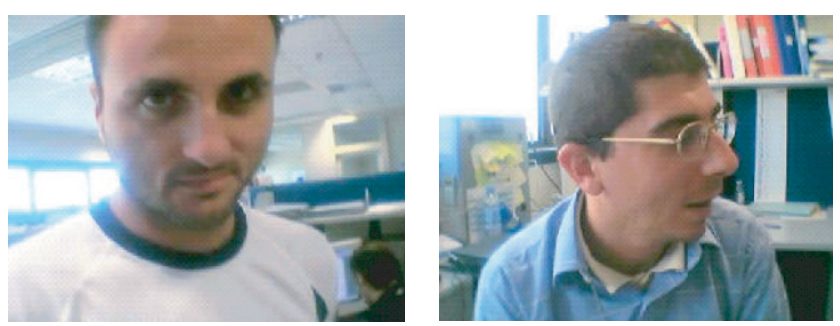

(b)

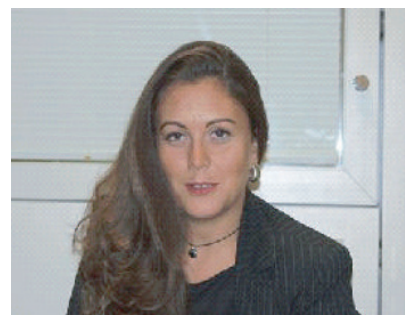

(d)

FIGURE 12: Experimental results: (a) original images acquired by Nokia 7650 VGA sensor compressed in JPEG format, (b) corrected output, (c) image acquired with CCD sensor (4.1 megapixels) Olympus E-10 camera, and (d) corrected output image.

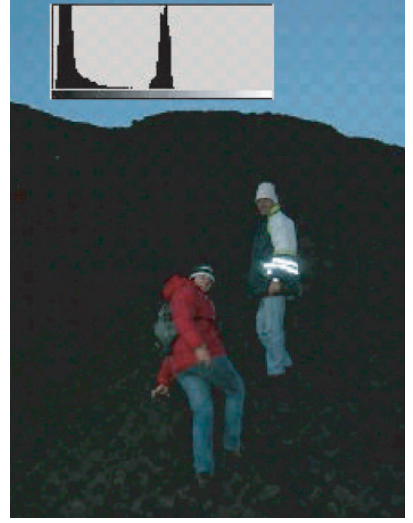

(a)

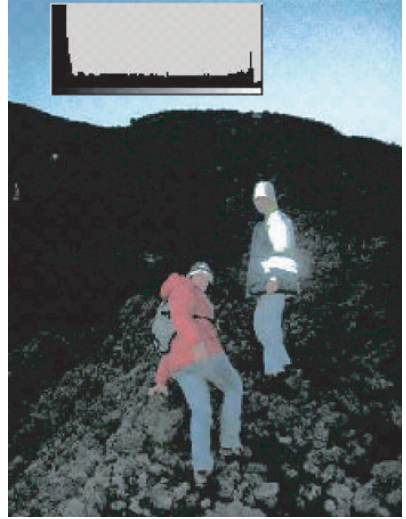

(b)

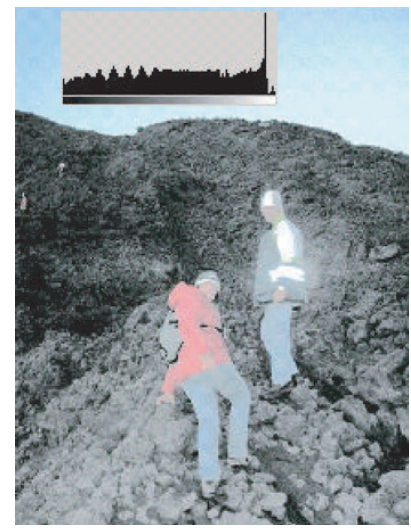

(c)

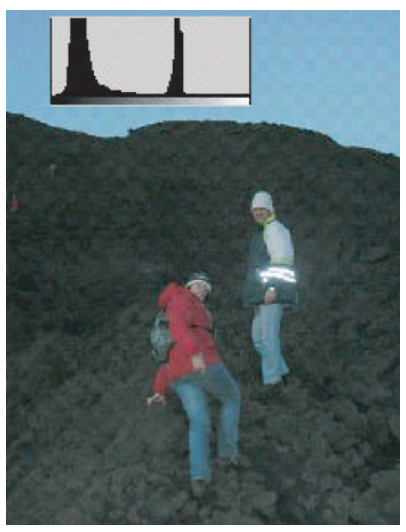

(d)

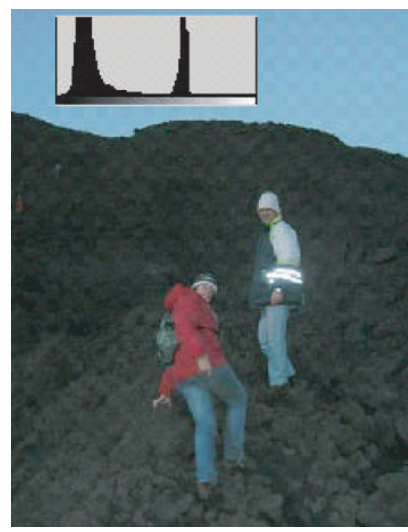

(e)

FIGURE 13: Experimental results with relative luminance histograms: (a) input image, (b) adaptive equalized image using the technique described in [9], (c) equalized image, (d) gamma correction output with fixed average value defined by the proposed method, and (e) proposed algorithm output. The selected image (a) has been captured by using an Olympus C120 camera. 


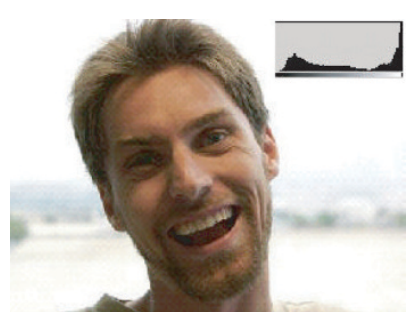

(a)

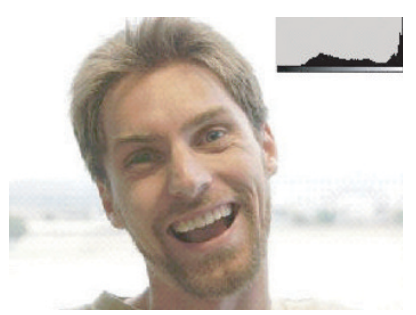

(d)

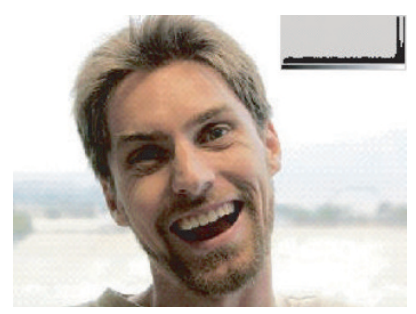

(b)

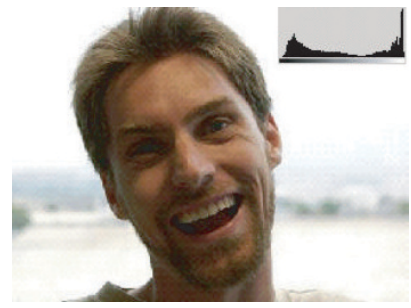

(e)

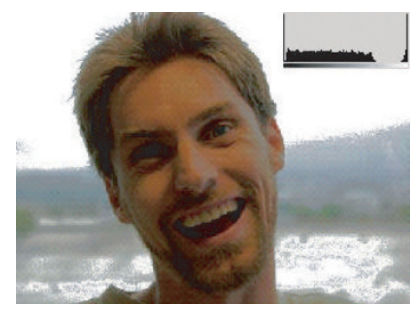

(c)

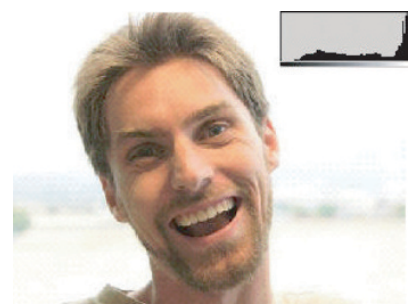

(f)

Figure 14: Experimental results with relative luminance histograms: (a) input image, (b) adaptive equalized image using the technique described in [9], (c) equalized image, (d) gamma correction output with fixed average value defined by the proposed method, (e) proposed algorithm forced without skin feature detection, and (f) proposed algorithm output. The selected image (a) has been captured by using an Olympus E10 camera.

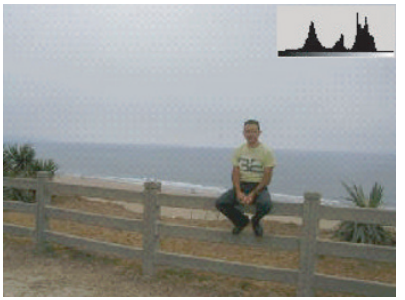

(a)

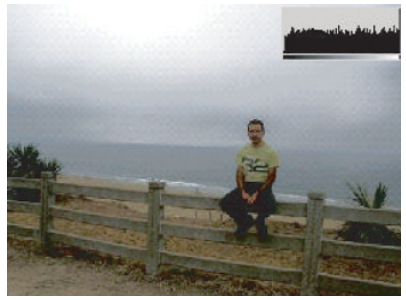

(b)

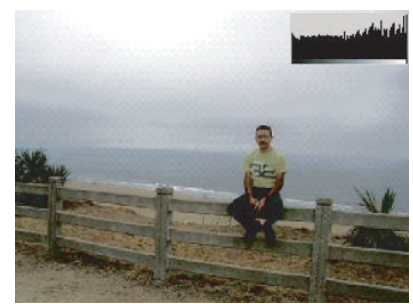

(c)

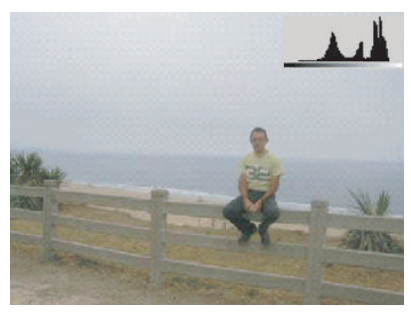

(d)

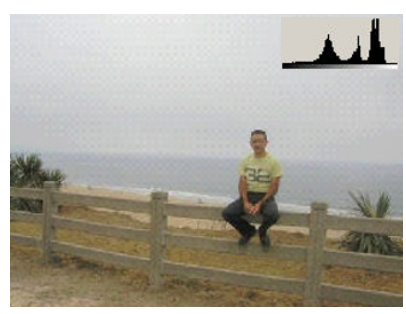

(e)

FIGURE 15: Experimental results with relative luminance histograms: (a) input image, (b) equalized image, (c) adaptive equalized image using the technique described in [9], (d) gamma correction output with fixed average value computed by the proposed method, and (e) proposed algorithm output. The selected image (a) has been captured by using an Olympus C40Z camera.

Almost all gamma-corrected images in Figures 13d, 14d, and $15 \mathrm{~d}$ contain excessive color desaturation.

Results show how often histogram equalization, that do not take into account images features, leads to excessive contrast enhancement while simple gamma correction leads to excessive color desaturation. Therefore the features analysis capability of the proposed algorithm permits contrast en- hancement taking into account some strong peculiarity of the input image.

\section{CONCLUSIONS}

A method for automatic exposure correction, improved by different feature extraction techniques, has been described. 
The approach is able to analyze the Bayer data captured by a CCD/CMOS sensor, or the corresponding color generated picture; once the skin key features have been identified, the algorithm adjusts the exposure level using a "camera response"-like function. The method can solve some of the typical drawbacks featured by handset devices due to poor optics, absence of flashgun, difficult scene lighting conditions, and so forth. The overall computation time needed to apply the proposed algorithm, is negligible, thus it is well suited for real-time applications. Experiments show the effectiveness of the techniques in both cases. Future works will investigate the use of curvelet transform for enhanced exposure correction [13].

\section{REFERENCES}

[1] S. Battiato, A. Castorina, and M. Mancuso, "High dynamic range imaging for digital still camera: an overview," Journal of Electronic Imaging, vol. 12, no. 3, pp. 459-469, 2003.

[2] A. Bosco, M. Mancuso, S. Battiato, and G. Spampinato, "Temporal noise reduction of Bayer matrixed video data," in Proc. IEEE International Conference on Multimedia and Expo (ICME '02), vol. 1, pp. 681-684, Lausanne, Switzerland, August 2002.

[3] M. Mancuso, A. Bosco, S. Battiato, and G. Spampinato, "Adaptive temporal filtering for CFA video sequences," in Proc. IEEE Advanced Concepts for Intelligent Vision Systems (ACIVS '02), pp. 19-24, Ghent University, Belgium, September 2002.

[4] G. Messina, S. Battiato, M. Mancuso, and A. Buemi, "Improving image resolution by adaptive back-projection correction techniques," IEEE Transactions on Consumer Electronics, vol. 48, no. 3, pp. 409-416, 2002.

[5] J. Holm, I. Tastl, L. Hanlon, and P. Hubel, "Color processing for digital photography," in Colour Engineering: Achieving Device Independent Colour, P. Green and L. MacDonald, Eds., John Wiley \& Sons, New York, NY, USA, June 2002.

[6] M. Mancuso and S. Battiato, "An introduction to the digital still camera technology," ST Journal of System Research, vol. 2, no. 2, pp. 1-9, 2001.

[7] R. C. Gonzalez and R. E. Woods, Digital Image Processing, Addison-Wesley, Reading, Mass, USA, 1993.

[8] J. A. Stark, "Adaptive image contrast enhancement using generalizations of histogram equalization," IEEE Trans. Image Processing, vol. 9, no. 5, pp. 889-896, 2000.

[9] Y. Wang, Q. Chen, and B. Zhang, "Image enhancement based on equal area dualistic sub-image histogram equalization method," IEEE Transactions on Consumer Electronics, vol. 45, no. 1, pp. 68-75, 1999.

[10] J. A. S. Centeno and V. Haertel, "An adaptive image enhancement algorithm," Pattern Recognition, vol. 30, no. 7, pp. 1183$1189,1997$.

[11] H. D. Cheng and H. Xu, "A novel fuzzy logic approach to contrast enhancement," Pattern Recognition, vol. 33, no. 5, pp. 809-819, 2000.

[12] J. Tang, E. Peli, and S. Acton, "Image enhancement using a contrast measure in the compressed domain," IEEE Signal Processing Letters, vol. 10, no. 10, pp. 289-292, 2003.

[13] J.-L. Starck, F. Murtagh, E. Candes, and D. L. Donoho, "Gray and color image contrast enhancement by the curvelet transform," IEEE Trans. Image Processing, vol. 12, no. 6, pp. 706$717,2003$.

[14] S. A. Bhukhanwala and T. V. Ramabadran, "Automated global enhancement of digitized photographs," IEEE Transactions on Consumer Electronics, vol. 40, no. 1, pp. 1-10, 1994.
[15] B. E. Bayer, “Color imaging array,” US Patent 3971 065,1976.

[16] J. Reichel and M. J. Nadenau, "How to measure arithmetic complexity of compression algorithms: a simple solution," in Proc. IEEE International Conference on Multimedia and Expo (ICME '00), vol. 3, pp. 1743-1746, New York, NY, USA, JulyAugust 2000.

[17] S. L. Phung, A. Bouzerdoum, and D. Chai, "A novel skin color model in $\mathrm{YCbCr}$ color space and its application to human face detection," in Proc. IEEE International Conference on Image Processing (ICIP '02), vol. 1, pp. 289-292, Rochester, NY, USA, September 2002.

[18] B. D. Zarit, B. J. Super, and F. K. H. Quek, "Comparison of five color models in skin pixel classification," in Proc. IEEE International Workshop on Recognition, Analysis, and Tracking of Faces and Gestures in Real-Time Systems (RATFG-RTS '99), pp. 58-63, Corfu, Greece, September 1999.

[19] J. Yang, W. Lu, and A. Waibel, "Skin-color modeling and adaptation," Tech. Rep. CMU-CS-97-146, School of Computer Science, Carnegie Mellon University, Pittsburgh, Pa, USA, 1997.

[20] Colour Sensor Evaluation Kit VV6501, STMicroelectronics, Edinburgh, www.st.com/stonline/products/applications/ consumer/ cmos_imaging/sensors/6501.htm.

[21] M. Soriano, B. Martinkauppi, S. Huovinen, and M. Laaksonen, "Skin color modeling under varying illumination conditions using the skin locus for selecting training pixels," in Proc. Workshop on Real-time Image Sequence Analysis (RISA '00), pp. 43-49, Oulu, Finland, August-September 2000.

[22] S. Mann, "Comparametric equations with practical applications in quantigraphic image processing," IEEE Trans. Image Processing, vol. 9, no. 8, pp. 1389-1406, 2000.

[23] S. Sakaue, M. Nakayama, A. Tamura, and S. Maruno, "Adaptive gamma processing of the video cameras for the expansion of the dynamic range," IEEE Transactions on Consumer Electronics, vol. 41, no. 3, pp. 555-562, 1995.

S. Battiato received the Ph.D. degree in 1999 in applied mathematics and computer science from Catania University. From 1999 to 2003 he was at STMicroelectronics in the Advanced System Technology (AST) Catania Laboratory with the Imaging Group. He is currently a Researcher And Teaching Assistant in the Department of Mathematic and Informatics at the University of Catania. His current research interests lie in the areas of digital image processing, pattern recognition, and computer vision. He acts as a reviewer for several leading international conferences and journals, and he is author of several papers and international patents.

A. Bosco was born in Catania, Italy, in 1972. He received the M.S. degree in computer science in 1997 from the University of Catania with a thesis in the field of image processing about tracking vehicles in video sequences. He joined STMicroelectronics in June 1999 as a System Engineer in the Digital Still Camera and Mobile Multimedia Group. Since then, he has been working on distortion artifacts of CMOS imagers and noise reduction, both for still pictures and video. His current activities deal with image quality enhancement and noise reduction. Some of his works have been patented and published in various papers in the image processing field. 
A. Castorina received his M.S. degree in computer science in 2000 from the University of Catania. His thesis is about watermarking algorithms for digital images. Since September 2000 he has been working in STMicroelectonics in the AST Digital Still Camera Group as System Engineer. His current activities include image enhancement and high dynamic range imaging.

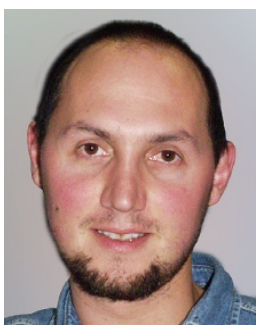

G. Messina received his M.S. degree in computer science in 2000 from the University of Catania. His thesis is about statistical methods for textures discrimination. Since March 2001 he has been working at STMicroelectronics in the Advanced System Technology (AST) Imaging Group as System Engineer. His current research interests are in the area of image processing, resolution enhancement, analysis-synthesis of tex-

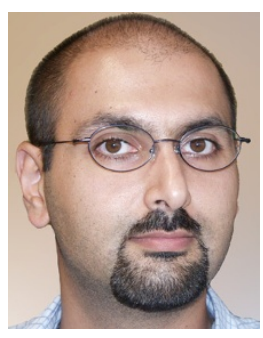
ture, and color interpolation. He is author of several papers and patents in image processing field. 